Profiling the autoantibody repertoire by serological antigen selection Peer-reviewed author version

SOMERS, Veerle; GOVARTS, Cindy; HELLINGS, Niels; Hupperts, R. \& STINISSEN, Piet (2005) Profiling the autoantibody repertoire by serological antigen selection. In: JOURNAL OF AUTOIMMUNITY, 25(3). p. 223-228.

DOI: 10.1016/j.jaut.2005.09.023

Handle: http://hdl.handle.net/1942/1002 


\title{
Profiling the autoantibody repertoire by Serological
} Antigen Selection

\author{
V. Somers ${ }^{1}$, C. Govarts ${ }^{1}$, N. Hellings ${ }^{1}$, R. Hupperts ${ }^{2}$ and P. Stinissen ${ }^{1}$ \\ ${ }^{1}$ Hasselt University, Biomedical Research Institute, and Transnationale Universiteit Limburg, \\ School of Life Sciences, Agoralaan, Building A, B-3590 Diepenbeek, Belgium \\ 2 Department of Neurology, Academical Hospital Maastricht, P.O. Box 5800, 6202 AZ \\ Maastricht, the Netherlands
}

* Corresponding author: Dr. Veerle Somers, Hasselt University, Biomedical Research Institute, and Transnationale Universiteit Limburg, School of Life Sciences, Agoralaan, Building A, B-3590 Diepenbeek, Belgium, Tel: +32-11269202, Fax: +32-11269209, Email: veerle.somers@luc.ac.be, Please note that as of June 15 this email address will change to veerle.somers@uhasselt.be 


\begin{abstract}
The identification of disease related autoantigens targeted by pathogenic $\mathrm{T}$ - and B-cell responses is crucial for the development of improved therapies for autoimmune diseases. To identify immunogenic targets recognized by the humoral immune response, we have recently applied a novel and powerful molecular approach, named 'Serological antigen selection'. This method involves the display of a cDNA expression library on filamentous phage and subsequent selection on patient Immunoglobulin G (IgG). In the present study, we have cloned a cDNA repertoire from a Multiple Sclerosis (MS) patient in pVI phage display vectors and performed selections on pooled MS cerebrospinal fluid samples (CSF) immobilized with anti-human IgG. To further streamline this procedure, we report an optimized SAS procedure in which we have successfully established methods for enrichment of MS-specific candidate antigens. In conclusion, the broad applicability of the SAS method makes it a highly promising method for investigating the autoimmune repertoire.
\end{abstract}

Keywords: Serological Antigen Selection, filamentous phage, cDNA display, Multiple Sclerosis, autoantibody repertoire, autoimmune diseases

Abbreviations: SAS, Serological Antigen Selection; pVI, bacteriophage coat protein 6 


\section{INTRODUCTION}

The identification of B and T cell epitopes is a crucial step for the understanding of the immune response mechanisms and their role in autoimmune diseases. Although some autoimmune diseases have been mainly considered as T-cell mediated diseases, the importance of autoreactive B cells has long been underestimated. However, the number of autoimmune diseases associated with the presence of autoantibodies directed against cells of the target tissue has been growing extensively over the past years $[1,2,3,4]$. Therefore, the role of B cells and antibodies has been re-evaluated in several autoimmune diseases such as multiple sclerosis.

Multiple sclerosis is a chronic, inflammatory disease of the central nervous system characterized by multifocal inflammation and destruction of myelin. Although the etiology is unknown, it is generally accepted that a misdirected immune response plays a crucial role in the pathogenesis of the disease. Several findings suggest an important role of B cells and autoantibodies in MS. In chronic MS lesions, B cells, plasma cells and antibodies have been demonstrated $[5,6]$. Analysis of the heavy chain repertoire of B cells in the central nervous system revealed a limited Immunoglobulin $\mathrm{G}(\mathrm{IgG})$ repertoire, which is consistent with a targeted immune response $[7,8,9,10]$. In addition, cerebrospinal fluid (CSF) of patients with clinically definite MS is characterized by the presence of oligoclonal antibodies (seen as oligoclonal bands), which is used as a diagnostic indicator of disease [11]. Accordingly, in the serum, where these bands are not observed using isoelectric focusing, the same antibodies should be either absent, or, if present, not quantitatively predominant.

The isolation of autoantigens recognized by autoantibodies present in e.g. sera from patients with autoimmune diseases has been traditionally carried out through time-consuming

processes. One of the first methods described used immunoprecipitation and immunoblotting to identify candidate autoantigens $[12,13]$. More recent procedures focused on the serological screening of cDNA libraries constructed with lambda phage vectors [14]. Although this procedure has led to successful applications in several autoimmune diseases $[15,16,17]$, the labor intensity and non-quantitative nature of the screening procedure have impeded the analysis of many different candidate autoantigens on multiple normal and patient sera.

During the past decade, filamentous phage display has been used to identify ligands for any type of ligate. Based on this system, random peptide libraries have been constructed and screened on different ligates such as monoclonal antibodies and even more complex 
patient sera $[18,19,20,21]$. However, the main disadvantage is that only antigen mimotopes are recovered by which further analysis is required to actually clone the serologically defined antigen. In multiple sclerosis, the use of random peptide libraries has mainly resulted in the isolation of candidate peptides which showed patient specific immunoreactivity $[22,23]$.

To fully explore the complex information present within the antibody repertoire of patients, we have recently applied a novel and powerful molecular approach, called 'Serological antigen selection'. This method involves the display of a cDNA expression library on filamentous phage by fusing the cDNA product to minor coat protein pVI [24]. The filamentous phage links the expressed and displayed cDNA product to the DNA, cloned into the phage genome. This allows the selection and enrichment of those cDNA products that interact with a chosen immobilized or labeled ligand, for example, patient IgG. We have successfully applied this method to molecularly define antigens recognized by the humoral immune system in colorectal cancer, and this gave rise to the isolation of a panel of candidate tumor antigens in colorectal cancer [25].

The present study reports the cloning and display of an MS cDNA library from the target tissue on filamentous phage and enrichment by affinity selection on MS patient CSF and serum IgG. By using the patient's autoantibody repertoire to identify those cDNA expression products or antigens that evoke an IgG immune response, interesting candidates can be identified for further study. To further streamline this procedure, we report an optimized SAS procedure in which we have successfully established methods for enrichment of MS-specific candidate antigens. A panel of antigenic cDNAs with an MS-related immunogenicity holds promise as a valuable tool in a diagnostic setting. 


\section{MATERIALS AND METHODS}

\section{Cloning of an MS cDNA library for pVI display}

A normalized cDNA library $\left(1.0 \times 10^{6}\right.$ primary recombinants $)$ derived from active chronic MS plaques, with varying degrees of demyelination and inflammatory activity (gift from Dr. Soares) was transferred into our phage cDNA display vectors, named pSPVIA, pSPVIB and pSPVIC, each encoding one of three reading frames. The normalized cDNA library was prepared following a procedure based on reassociation kinetics [26]. To construct the cDNA display libraries, plasmid DNA was prepared from the normalized MS cDNA library and further digested with EcoRI and NotI. DNA fragments were gel purified (GFX gel band purification kit, Amersham Biosciences) and ligated into the three vectors pSPVIA, pSPVIB and pSPVIC digested with EcoRI and NotI. The ligation mixtures were used to transform Escherichia coli TG1 cells by electroporation to obtain libraries MS-pSPVIA, MSpSPVIB and MS-pSPVIC of sizes $9.0 \times 10^{6}, 1.0 \times 10^{6}$ and $1.0 \times 10^{6}$ independent clones respectively.

\section{CSF/Sera}

Cerebrospinal fluid samples and sera were obtained from MS patients and patients with other inflammatory (meningitis, polyneuropathy) and non-inflammatory neurological disorders (hernia, epilepsy, dementia, ...). In addition, sera were collected from healthy volunteers. CSF and serum samples were stored at $-80^{\circ} \mathrm{C}$ after collection. CSF and serum samples used for the selection procedure were absorbed against E. Coli and phage antibodies by repeated passage through columns of Sepharose 6MB (Pharmacia, Uppsala, Sweden) coupled to lysates of E. Coli Y1090 and bacteriophage-infected E. Coli XL1Blue. Following absorption steps, aliquots from CSF and serum samples were prepared in $0.2 \%(\mathrm{w} / \mathrm{v})$ skimmed milk powder in $1 \mathrm{x}$ TBS $(50 \mathrm{mM}$ Tris- $\mathrm{HCl}(\mathrm{pH} 7.9)$ and $150 \mathrm{mM} \mathrm{NaCl})$ and stored at $-20^{\circ} \mathrm{C}$.

\section{Selection of phage pVI displayed cDNA repertoires}

CSF samples of 10 untreated relapsing remitting (RR) MS patients (8 women, 2 men, age 32-51, EDSS 0-3.5) were pooled and used for affinity selections. Selections were performed as described before with slight adaptations [25]. In brief, immunotubes (Nunc, 
Roskilde, Denmark) were coated with rabbit anti-human IgG (Dako, Glostrup, Denmark) at a concentration of $10 \mu \mathrm{g} / \mathrm{ml}$ in coating buffer $(0.1 \mathrm{M}$ sodium hydrogen carbonate $\mathrm{pH} 9.6)$ for 2 hours at $37^{\circ} \mathrm{C}$. After washing the immunotube twice with phosphate-buffered saline/Tween 20 (PBST: $50 \mathrm{mM}$ Tris, $150 \mathrm{mM} \mathrm{NaCl}, \mathrm{PH}$ 7.5, 0.1\% Tween $20(\mathrm{w} / \mathrm{v})$ ) and twice with PBS, the tubes were blocked for 2 hours at room temperature (RT) with 2\% MPBS $(2 \% \mathrm{w} / \mathrm{v}$ milk powder in PBS). Phage were prepared from each library MS-pSPVIA, MS-pSPVI6B and MSpSPVIC as described previously [27]. Approximately equal numbers of phage derived from each phage library $\left( \pm 2 \times 10^{12} \mathrm{pfu}\right)$ were added to pooled CSF (1:5 diluted in $4 \%$ MPBS) and incubated in a glass tube for 1.5 hour at RT on a rotating platform. After washing the coated immunotube twice with PBST and twice with PBS, the preincubated CSF and phage mix was transferred to the coated immunotube and incubated for 30 minutes on a rotating platform and 120 minutes standing at RT. Tubes were then washed 20 times with PBST and 20 times with PBS to remove non-binding phage. Binding phage were eluted with $100 \mathrm{mM}$ triethylamine and neutralized with $1 \mathrm{M}$ Tris $\mathrm{HCl}$ as described before [25]. E. Coli TG1 cells were infected with input and output phage and plated on $2 \times \mathrm{TY}$ agar plates containing ampicillin and glucose (16 g/l bacto-tryptone, $10 \mathrm{~g} / 1$ yeast extract, $5 \mathrm{~g} / 1 \mathrm{NaCl}, 15 \mathrm{~g}$ bacto-agar/1, ampicillin at $100 \mu \mathrm{g} / \mathrm{ml}$ and glucose at $2 \% \mathrm{w} / \mathrm{v}$ ) at each round of selection. Resultant colonies were scraped and phage were rescued for further rounds of affinity selections. To monitor enrichment of specific clones, input and output phage from each round of selection were titrated and the ratio of output/input phage was determined.

\section{Depletion strategies of non-specific clones}

To enrich for selection of cDNA products binding to IgGs specifically associated with MS, alternative selection strategies on MS patient material and normal samples were tested. After a first selection round on a pool of 10 RR MS sera, a depletion strategy was developed by using a pool of 10 age-matched normal sera. Selections on the pool of MS sera (1/100 diluted in 4\% MPBS) were performed as described above. After this first selection round, phage were rescued and used for selection on IgGs present in the pool of age-matched normal sera (1/100 diluted in 4\% MPBS). After preincubation for 1.5 hour at RT on a rotating platform, the mixture was transferred to the coated immunotube for 30 minutes on a rotating platform and 120 minutes standing at RT, the non-bound phage was now recovered and used for infection of E. Coli TG1 cells. Phage were again rescued as described and the selection 
process of alternative selections on MS patient and normal sera was repeated. The ratio of output/input phage was determined after each selection round.

\section{Polymerase chain reaction (PCR) amplification and fingerprinting}

After several rounds of selection, individual colonies were selected and the insert size was determined with forward primer (5'-CTC TCT GTA AAG GCT GC-3') and reverse primer (5'-CGC CAG GGT TTT CCC AGT CAC GAC-3') which flank the EcoRI and NotI cloning sites of the vector, respectively. Briefly, colonies were subjected to 35 cycles (20", $94^{\circ} \mathrm{C}, 20^{\prime} 55^{\circ} \mathrm{C}, 40^{\prime}, 72^{\circ} \mathrm{C}$ ) of PCR amplification after an initial denaturation step for 5 ' at $95^{\circ} \mathrm{C}$ in a DNA Thermal Cycler (Perkin-Elmer Cetus, Norwalk, CT). The amplification products were analysed by electrophoresis on a $1.0 \%$ agarose gel to confirm the presence of a cDNA insert. After amplification, $10 \mu \mathrm{l}$ of the amplification products was used for fingerprinting analysis by incubating the amplification mixture with $5 \mathrm{U}$ of the restriction enzyme BstNI (Roche Diagnostics, Germany) for $2 \mathrm{hrs}$ at $37^{\circ} \mathrm{C}$. Products after restriction enzyme digestion were analyzed on a $2 \%$ agarose gel.

\section{ELISA of ligand displaying phage}

96-well flat-bottomed microtiter plates (Falcon) were coated overnight at $4{ }^{\circ} \mathrm{C}$ with $200 \mu \mathrm{l}$ of rabbit-anti human $\operatorname{IgG}$ (Dako), $10 \mu \mathrm{g} / \mathrm{ml}$ in coating buffer $(0.1 \mathrm{M}$ sodium hydrogen carbonate $\mathrm{pH} 9.6)$ and blocked with $200 \mu \mathrm{l}$ of $2 \%$ MPBS ( $2 \% \mathrm{w} / \mathrm{v}$ milk powder in PBS) for 1 hour at RT. For the ELISA screening on human CSF, a dilution of 1:5 (in 4\% MPBS) was used for the pool of 10 human CSF (each individual CSF was diluted 1:50) and $50 \mu$ l diluted CSF was used for preincubation with $100 \mu$ PEG-purified phage $\left(10^{10}\right.$ phages per well $)$ in a 96-well round-bottomed 96-well plate (Costar). For the screening on individual CSF, a dilution of 1:3 (in 4\% MPBS) was used. Both absorbed and non-absorbed patient CSF samples were used in ELISA experiments.

Phage particles were allowed to bind to patient CSF for 1 hour at $37^{\circ} \mathrm{C}$ and 30 minutes shaking at RT. After washing 3 times with PBSTween (0.1\% Tween 20) and 3 times with PBS, the preincubated CSF plus phage mixture was transferred to the coated plate for 1 hour at $37^{\circ} \mathrm{C}$ and 30 minutes shaking at RT. After washing, $150 \mu \mathrm{l}$ of a peroxidase conjugated anti-M13 monoclonal (Amersham/Pharmacia/Biotech), diluted 1:5,000 in 2\% MPBS (2\% w/v milk powder in PBS) was incubated for 1 hour shaking at RT. After washing the plates $3 \mathrm{x}$ 
with PBSTween and $3 x$ with PBS, $130 \mu \mathrm{l}$ of a 3,3',5,5' tetramethyl-benzidine dihydrochloride (TMB) chromogen solution $(10 \mathrm{mg} / \mathrm{ml})$ was added and the color development stopped with $65 \mu \mathrm{l} /$ well $2 \mathrm{M} \mathrm{H}_{2} \mathrm{SO}_{4}$. The plates were read at $450 \mathrm{~nm}$ in a BIORAD Novapath microplate reader. 


\section{RESULTS}

\section{Construction and enrichment of a phage displayed MS cDNA library with MS CSF}

A normalized cDNA library derived from active, chronic MS plaques, with varying degrees of demyelination and inflammatory activity, which was originally cloned into the pT7T3-Pac vector, was cloned into the pVI display vectors in 3 reading frames. A total library size of $1.1 \times 10^{7}$ colony forming units (cfu) was obtained. Analysis of 50 random clones from each library indicated insert sizes of the MS-cDNA repertoire ranging from $500 \mathrm{bp}$ to $2.5 \mathrm{~kb}$.

CSF samples from 10 RR MS patients were used for selections against a polyclonal rabbit anti-human IgG. We decided to pool patient material for the selection procedure because previous applications using peptide libraries resulted in the identification of clones which showed patient specific reactivity [22,23]. The procedure of 'Serological Antigen Selection' (SAS) is depicted in Figure 1. The selection procedure involves a pre-incubation step of the CSF pool with equal numbers of phage derived from each of the three displayed cDNA repertoires. IgG antibodies present in MS patient CSF bind to MS specific epitopes expressed on the phage surface. During this solution-based hybridization, high affinity interactions between antibody and antigen occur. The pre-incubated phage and CSF mixture is transferred to a solid phase for capture of phage cDNA-IgG complexes onto the surface. IgGs present in this mixture will adhere to the coating antibody while non-bounded phage are washed away. Bounded phage were eluted and used for a next amplification and selection step.

The results of affinity selection of the pVI phage displayed MS cDNA library on the pool of MS patient CSF are presented in Table 1. Following rescue of the phage clones after each of 4 rounds of selection, enrichment of phage could be seen reflected in an increase of the ratio output to input phage titer. Fingerprint analysis of the clones from the fourth round of selection showed that many different cDNA products had been selected, which also indicated that selective enrichment had occurred (data not shown). Although many cDNA products were successfully identified, a relatively high number $(>50 \%)$ of cDNAs were retrieved that encoded for IgG transcripts. These originated from B cells present in the MS brain used to construct the primary cDNA library. The insert sizes of the genes ranged from 600 to $2500 \mathrm{bp}$ and remained constant during selection cycles. 


\section{Results from depletion strategies}

In order to enhance the selection of cDNA products binding to IgGs specifically associated with MS, an optimization strategy was designed including alternative selections on MS patient material and normal samples. This method was illustrated using MS patient and normal sera. By repeated selections on MS patient sera and normal sera, the enrichment of cDNA clones encoding for IgG transcripts was dramatically decreased to $>50 \%$ of that without depleting steps (Table 2). Together with the IgG transcripts, a high number of phage clones binding to normal sera was retrieved in the depletion step, thereby increasing the identification of clones related to MS patient sera (data not shown). This improved approach allows the identification of candidate MS clones recognized by IgGs present in sera of MS patients (manuscript in preparation).

\section{Phage ELISA screening of antigens retrieved by the MS CSF pool}

In the first selection experiment we used pooled CSF from RR MS patients as selector. Four cycles of selection-amplification were performed and the efficiency of the selection was monitored by measuring the reactivity of the CSF pool to the enriched phage. Positive clones could be detected after the 3rd round of selection, with an increase in reactivity to the enriched phage by the CSF pool in the 4rd round of selection (data not shown). The unselected library and empty phage were used as controls.

To determine the frequency of the antibody response in the individual CSF samples comprising the MS CSF pool used for the selection procedure, a phage ELISA procedure was performed on the individual patient CSF samples. Figure 2 presents the results of the immunoreactivity of the 10 individual CSF samples and the CSF pool tested for 2 antigenic cDNAs identified. Reactivity to clone A1 and A2 was found in 2 of 10 individual CSF tested, respectively, while both clones were negative on $10 \mathrm{CSF}$ from patients with noninflammatory and other inflammatory neurological disorders tested (data not shown). Highly positive ELISA signals were obtained, with consistent background signals (reactivity to empty phage). The reactivity of the CSF pool was used as a positive control. The signal on individual CSF samples was much higher than that obtained on the CSF pool which was consistent with the dilution of the antigen specific immunoglobulins in the CSF pool. The threshold line of the background values was set at twice the mean of the background values. Both absorbed and non-absorbed patient CSF samples were used in ELISA experiments. No 
reduction in CSF titers was seen after absorbing CSF against phage and bacterial proteins with no differences in background signals of unreactive CSF samples.

To determine whether the cDNA clones from the selection of pooled MS patient CSF showed MS-related immunoreactivity, we are currently analyzing the frequency of the antibody responses in a large group of CSF samples of other MS patients not used for the selection procedure and CSF samples of patients with non-inflammatory and other inflammatory neurological disorders. 


\section{DISCUSSION}

Despite the apparent diversity of proteins that can be displayed on filamentous phage, the generation of representative cDNA libraries and selections on complex patient material has presented a substantial challenge. To apply this technology to autoimmune diseases like MS, optimal conditions for technology design and handling of patient material are inevitable. Both vector design and the method of library construction have important implication for the quality and diversity of the repertoire that can be displayed. Firstly, cDNA repertoires should be cloned in all reading frames and in a defined orientation. Secondly, selections under conditions of low selective pressure require a highly stable vector system. To further permit the isolation of very rare transcripts, a cDNA library size containing at least $10^{6}$ independent clones is necessary, but even larger repertoires are required when fragment repertoires are constructed. In addition, the presence of IgG transcripts, which hamper the identification of relevant candidate autoantigens, should be overcome by optimizing depleting strategies.

The present study clearly demonstrates that selection of phage-cDNAs on MS patient CSF and sera provides a fast molecular procedure for analyzing the humoral immune response. In addition, using SAS in MS patients, we have optimized the selection procedure, which allows the identification of possible candidate autoantigens by first depleting for the presence of IgG transcripts.

The cloning and selection of an MS cDNA library displayed as fusion to the Cterminus of the minor coat protein VI proved to be successful. In contrast to bacteriophage coat protein 3 (pIII) or 8 (pVIII), the phage coat protein pVI is not known to be involved in infection and has the characteristic that the C-terminus rather than the $\mathrm{N}$-terminus is believed to be surface-exposed [28]. Therefore, the possible advantage of $\mathrm{pVI}$ for the display of cDNA repertoires is that the presence of stop codons does not prevent display. Furthermore, the use of a normalized cDNA library of $10^{6}$ primary recombinants, directionally cloned in all 3 reading frames, has proven to give a representative library size.

To further improve the diversity of the library, one may perform selections on a fragmented cDNA expression library. It may be beneficial to express short truncated cDNA fragments as these may be more efficiently expressed and less toxic to the bacteria. A recent study on the comparison of full-length and fragmented libraries has demonstrated the facilitated display of the cytoplasmic domain of the Fc gamma receptor IIB in comparison 
with the full-length product of this receptor [29]. It should, however, be stated that fragment libraries need to be significantly larger in order to encompass all possible diversity.

Fingerprint analysis of randomly picked clones after cloning and affinity selection on MS patient CSF confirmed the diversity of the library and demonstrated clear distribution of the various insert sizes. The insert sizes of the clones ranged from 600-2500 bp, which corresponded to the insert size distribution of the original library, denoting no loss of gene sequences after cloning and affinity selection.

We chose to optimize a depletion strategy to diminish problems with the presence of IgG transcripts. Although previous publications including our own reported the use of a system in which the use of cell lines for construction of the cDNA libraries were described $[30],[25]$, we now report the use of a cDNA library from the target tissue and the successful selection on patient CSF. This phage display procedure provides several advantages over serological screening of cDNA libraries constructed with lambda phage vectors. First, by preincubating a cDNA library in a solution phase, denaturation of proteins displayed on the surface of the phage is avoided. This might allow the interaction of antibody with conformational epitopes that are recognized by humoral responses in autoimmune diseases. Furthermore, by repeated cycles of selection and amplification, immunoreactive cDNA products displayed on the surface of phage have been enriched. Using this selection procedure, even the lowest titer immune response would be expected to eventually recover the specific antigen.

The phage-ELISA screening system established in this study has been optimized after testing different MS and control CSF with specificity for different antigens. The use of different antigens prepared as purified phage and empty phage provide critical internal specificity controls that immediately eliminate polyreactive samples. In addition, the finding of differences in optical density signals demonstrates the quantitative nature of the ELISA procedure.

The greater simplicity and speed of this serological assay allows the rapid screening of other disease-related and autoimmune CSF and sera together with a control group consisting of healthy individuals which have been age/sex matched. These characteristics make the phage display method far superior over the SEREX screening procedure. In future, this enables high-throughput serological immunome mapping with the use of age-matched databases and autoimmune databases. Correlations may be made for MS type versus 
immunogenicity and MS grading versus immunogenicity. Finally, to enable the characterization of multiple clones in a fast and cost-effective way, high-throughput screening is required, by which the need for cDNA protein screening (arrays) is inevitable.

In conclusion, Serological Antigen Selection i) is amenable to high throughput analysis, ii) yields a molecular profile of antibody-inducing antigens, and iii) may identify disease-related (auto)antigens, early detection of changes in immune responses due to infection, and possibly even surrogate markers for disease of unknown etiology. 
Acknowledgments: This study was supported by a grant from the Bijzonder Onderzoeksfonds Limburg (BOF) of the Hasselt University and by the transnational University Limburg (tUL). We thank Dr. Soares for providing the MS cDNA library and Dr. Medaer for collection of the MS CSF samples. 
Figure 1. Procedure of $\boldsymbol{S A S}$. The $S A S$ procedure is depicted for enrichment of a cDNA display library on CSF. a) A phage-displayed MS-cDNA repertoire is preincubated with patient CSF antibodies. b) MS-specific antigens displayed on phage (black) bind to MS antigen-specific patient IgG (black). c) Phage antigen-IgG complexes (black) are captured on a surface coated with polyclonal anti-human IgG (checked). d) Nonrelevant phage are washed away, and CSF IgG-specific phage are eluted. e) Selected phage are used for reinfection of bacteria. f) Selected phage are amplified and used for further rounds of selection. 
Figure 2. Reactivity to 2 selected antigens by both individual MS patient CSF and the CSF pool. The optical densities of 10 individual MS patient CSF to clone A1 and A2 are presented. Average values (OD $450 \mathrm{~nm}$ ) from 2 independent experiments have been determined. Both selected antigens were negative on 10 CSF from patients with noninflammatory and other inflammatory neurological disorders. 


\section{References}

1. Archelos J.J., Storch M.K., Hartung H.P. 2000. The role of B cells and autoantibodies in multiple sclerosis. Ann.Neurol. 47: 694-706

2. Leslie D., Lipsky P., Notkins A.L. 2001. Autoantibodies as predictors of disease. J.Clin.Invest 108: 1417-1422

3. Sherer Y., Gorstein A., Fritzler M.J., Shoenfeld Y. 2004. Autoantibody explosion in systemic lupus erythematosus: more than 100 different antibodies found in SLE patients. Semin.Arthritis Rheum. 34: 501-537

4. Rott S., Mrowietz U. 2005. Recent developments in the use of biologics in psoriasis and autoimmune disorders. The role of autoantibodies. BMJ 330: 716-720

5. Genain C.P., Cannella B., Hauser S.L., Raine C.S. 1999. Identification of autoantibodies associated with myelin damage in multiple sclerosis. Nat.Med. 5: $170-175$

6. Lucchinetti C., Bruck W., Parisi J., Scheithauer B., Rodriguez M., Lassmann H. 2000. Heterogeneity of multiple sclerosis lesions: implications for the pathogenesis of demyelination. Ann.Neurol. 47: 707-717

7. Baranzini S.E., Jeong M.C., Butunoi C., Murray R.S., Bernard C.C., Oksenberg J.R. 1999. B cell repertoire diversity and clonal expansion in multiple sclerosis brain lesions. J.Immunol. 163: 5133-5144

8. Colombo M., Dono M., Gazzola P., Roncella S., Valetto A., Chiorazzi N., Mancardi G.L., Ferrarini M. Accumulation of clonally related B lymphocytes in the cerebrospinal fluid of multiple sclerosis patients.

9. Owens G.P., Kraus H., Burgoon M.P., Smith-Jensen T., Devlin M.E., Gilden D.H. 1998. Restricted use of VH4 germline segments in an acute multiple sclerosis brain. Ann.Neurol. 43: 236-243

10. Qin Y., Duquette P., Zhang Y., Talbot P., Poole R., Antel J. 1998. Clonal expansion and somatic hypermutation of $\mathrm{V}(\mathrm{H})$ genes of $\mathrm{B}$ cells from cerebrospinal fluid in multiple sclerosis. J.Clin.Invest 102: 1045-1050

11. Poser C.M., Paty D.W., Scheinberg L., McDonald W.I., Davis F.A., Ebers G.C., Johnson K.P., Sibley W.A., Silberberg D.H., Tourtellotte W.W. 1983. New diagnostic criteria for multiple sclerosis: guidelines for research protocols. Ann.Neurol. 13: 227-231

12. Friedman J., Buskirk D., Marino L.J., Jr., Zabriskie J.B. 1987. The detection of brain antigens within the circulating immune complexes of patients with multiple sclerosis. J.Neuroimmunol. 14: 1-17 
13. Peuchen S., Gruemer H.D., DeVries G.H. 1992. Identification of a 58-kDa antigen with increased immunoreactivity in the cerebella of multiple sclerosis patients. J.Neuroimmunol. 41: 71-79

14. Krebs P., Kurrer M., Sahin U.T.Ö.L.B. 2003. Autoimmunity seen through the SEREX-scope. Autoimmunity Reviews 2: 339-345

15. Schmits R., Kubuschok B., Schuster S., Preuss K.D., Pfreundschuh M. 2002. Analysis of the B cell repertoire against autoantigens in patients with giant cell arteritis and polymyalgia rheumatica. Clin.Exp.Immunol. 127: 379-385

16. Jeoung D.I., Bong L.E., Lee S., Lim Y., Lee D.Y., Kim J., Kim H.Y., Wook S.Y. 2002. Autoantibody to DNA binding protein B as a novel serologic marker in systemic sclerosis. Biochem.Biophys.Res.Commun. 299: 549-554

17. Lim Y., Lee D.Y., Lee S., Park S.Y., Kim J., Cho B., Lee H., Kim H.Y., Lee E., Song Y.W., Jeoung D.I. 2002. Identification of autoantibodies associated with systemic lupus erythematosus. Biochem.Biophys.Res.Commun. 295: 119-124

18. Motti C., Nuzzo M., Meola A., Galfre G., Felici F., Cortese R., Nicosia A., Monaci P. 1994. Recognition by human sera and immunogenicity of HBsAg mimotopes selected from an M13 phage display library. Gene 146: 191-8

19. Cortese R., Monaci P., Luzzago A., Santini C., Bartoli F., Cortese I., Fortugno P., Galfre G., Nicosia A., Felici F. 1996. Selection of biologically active peptides by phage display of random peptide libraries. Curr Opin Biotechnol 7: 616-21

20. Dunn I.S. 1996. Phage display of proteins. Curr Opin Biotechnol 7: 547-53

21. Folgori A., Tafi R., Meola A., Felici F., Galfre G., Cortese R., Monaci P., Nicosia A. 1994. A general strategy to identify mimotopes of pathological antigens using only random peptide libraries and human sera. Embo J 13: 2236-43

22. Cortese I., Tafi R., Grimaldi L.M., Martino G., Nicosia A., Cortese R. 1996. Identification of peptides specific for cerebrospinal fluid antibodies in multiple sclerosis by using phage libraries. Proc.Natl.Acad.Sci.U.S.A 93: 11063-11067

23. Cortese I., Capone S., Luchetti S., Grimaldi L.M., Nicosia A., Cortese R. 1998. CSFenriched antibodies do not share specificities among MS patients. Mult.Scler. 4: $118-123$

24. Hufton S.E., Moerkerk P.T., Meulemans E.V., de Bruine A., Arends J.W., Hoogenboom H.R. 1999. Phage display of cDNA repertoires: the pVI display system and its applications for the selection of immunogenic ligands. $J$ Immunol Methods 231: 39-51

25. Somers V.A., Brandwijk R.J., Joosten B., Moerkerk P.T., Arends J.W., Menheere P., Pieterse W.O., Claessen A., Scheper R.J., Hoogenboom H.R., Hufton S.E. 2002. A panel of candidate tumor antigens in colorectal cancer revealed by the 
serological selection of a phage displayed cDNA expression library. J.Immunol. 169: 2772-2780

26. Soares M.B., Bonaldo M.F., Jelene P., Su L., Lawton L., Efstratiadis A. 1994. Construction and characterization of a normalized cDNA library. Proc.Natl.Acad.Sci.U.S.A 91: 9228-9232

27. Marks J.D., Hoogenboom H.R., Bonnert T.P., McCafferty J., Griffiths A.D., Winter G. 1991. By-passing immunization. Human antibodies from V-gene libraries displayed on phage. J Mol Biol 222: 581-97

28. Jespers L.S., Messens J.H., De Keyser A., Eeckhout D., Van den Brande I., Gansemans Y.G., Lauwereys M.J., Vlasuk G.P., Stanssens P.E. 1995. Surface expression and ligand-based selection of cDNAs fused to filamentous phage gene VI. Biotechnology (N Y) 13: 378-82

29. Cochrane D., Webster C., Masih G., McCafferty J. 2000. Identification of natural ligands for $\mathrm{SH} 2$ domains from a phage display cDNA library. Mol Biol 297: 89-97

30. Archelos J.J., Trotter J., Previtali S., Weissbrich B., Toyka K.V., Hartung H.P. 1998. Isolation and characterization of an oligodendrocyte precursor-derived B-cell epitope in multiple sclerosis. Ann.Neurol. 43: 15-24 


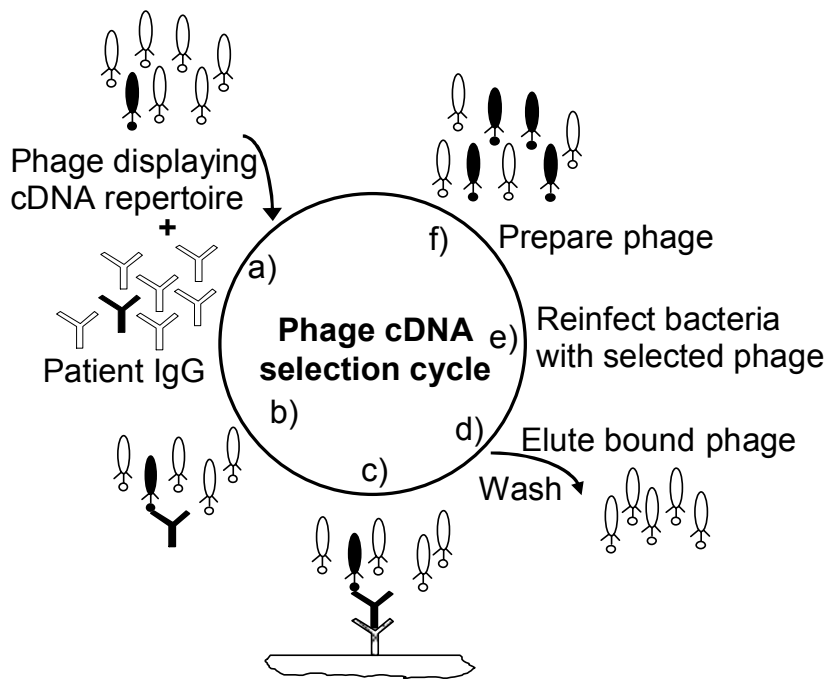

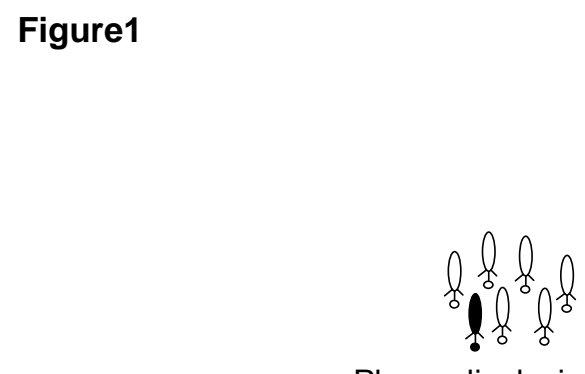
cDNA repertoire

Y a)

Y Phage cDNA e) Reinfect bacteria 


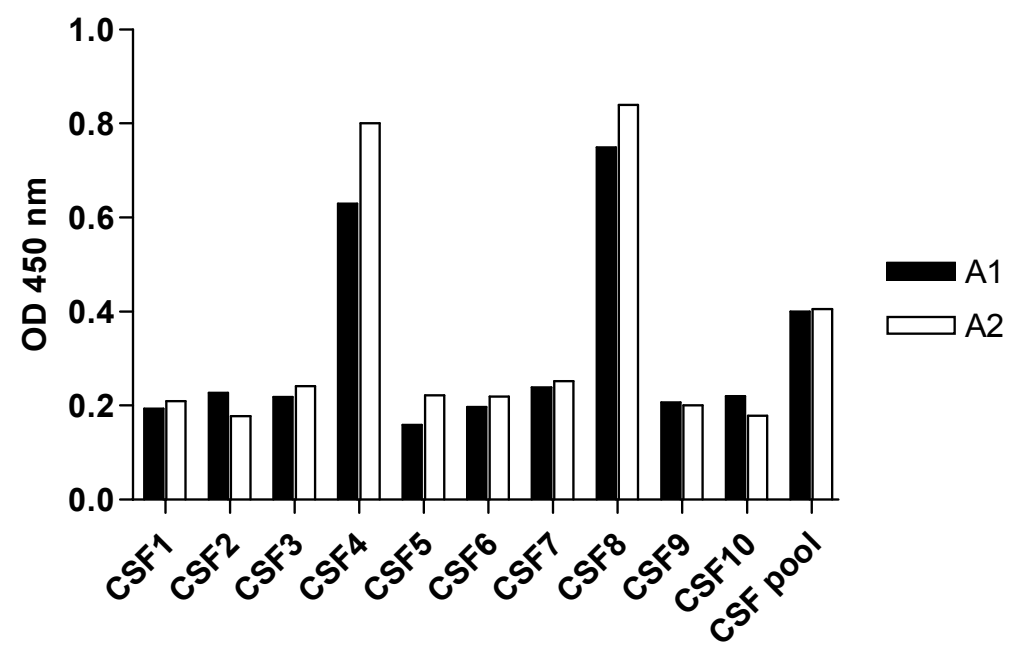




\section{Table 1. Selection of pVI phage displayed MS cDNA library on MS patient CSF}

$\begin{array}{llll}\text { Round } & \text { Input }^{\mathrm{a}} & \text { Output }^{\mathrm{a}} & \text { Ratio }(\mathrm{O} / \mathrm{I})^{\mathrm{b}} \\ 1 & 0.8 \times 10^{13} & 1.3 \times 10^{7} & 1.6 \times 10^{-6} \\ 2 & 0.6 \times 10^{13} & 1.6 \times 10^{6} & 2.6 \times 10^{-6} \\ 3 & 3.6 \times 10^{13} & 4.4 \times 10^{8} & 1.2 \times 10^{-5} \\ 4 & 7.0 \times 10^{13} & 1.5 \times 10^{9} & 2.1 \times 10^{-5}\end{array}$

a) The titration of input (I) and output (O) phage is presented as $\mathrm{cfu} / \mathrm{ml}$

b) Ratio (O/I) 


\section{Table 2. Selection of pVI phage displayed MS cDNA library on MS patient CSF}

$\begin{array}{lllll}\text { Round } & \text { Input }^{\mathrm{a}} & \text { Output }^{\mathrm{a}} & \text { Ratio }(\mathrm{O} / \mathrm{I})^{\mathrm{b}} & \% \mathrm{IgG}^{\mathrm{c}} \\ 1 \mathrm{MS} & 2.4 \times 10^{13} & 2.0 \times 10^{5} & 8.3 \times 10^{-9} & 0 \% \\ 2 \text { normal } & 0.7 \times 10^{13} & 2.7 \times 10^{12(\mathrm{~d})} & 3.8 \times 10^{-1} & 0 \% \\ 3 \mathrm{MS} & 0.5 \times 10^{13} & 1.6 \times 10^{6} & 3.2 \times 10^{-7} & 51,9 \% \\ 4 \text { normal } & 3.9 \times 10^{13} & 4.5 \times 10^{12 \mathrm{~d}} & 1.1 \times 10^{-1} & 18,9 \%\end{array}$

a) The titration of input (I) and output (O) phage is presented as cfu/ml

b) Ratio (O/I)

c) The percentage of IgG transcripts was determined by PCR and fingerprinting, and the percentage of IgG encoding transcripts is indicated

d) The results of the non-bound phage are presented 